\title{
Classification of bound exciton complexes in bulk ZnO by magnetophotoluminescence spectroscopy
}

\author{
L. Ding, ${ }^{1, a)}$ B. K. Li, ${ }^{1}$ H. T. He, ${ }^{1}$ W. K. Ge, ${ }^{1, b)}$ J. N. Wang, ${ }^{1, c)}$ J. Q. Ning, ${ }^{2}$ X. M. Dai, ${ }^{2}$ \\ C. C. Ling, ${ }^{2}$ and S. J. $\mathrm{Xu}^{2}$ \\ ${ }^{1}$ Department of Physics, Hong Kong University of Science and Technology, Clear Water Bay, Hong Kong, \\ China \\ ${ }^{2}$ Department of Physics and HKU-CAS Joint Laboratory on New Materials, The University of Hong Kong, \\ Pokfulam Road, Hong Kong, China
}

(Received 23 November 2008; accepted 22 January 2009; published online 9 March 2009)

\begin{abstract}
A series of bound exciton transitions of a bulk $\mathrm{ZnO}$ sample has been studied by high resolution magnetophotoluminescence spectra. Ten sharp intense bound exciton emissions are classified into three groups according to their dependences on magnetic field, angle, and circular polarization of the emission lines. The charge states of the defect centers and exciton types are identified. The effective $g$ factors of the electrons and holes are determined. (C) 2009 American Institute of Physics.
\end{abstract}

[DOI: $10.1063 / 1.3087762]$

\section{INTRODUCTION}

$\mathrm{ZnO}$ as a promising material for optoelectronic applications ${ }^{1}$ attracts more and more studies on its fundamental properties. In wurtzite $\mathrm{ZnO}$, free electrons occupy the $\Gamma_{7}$ conduction band and free holes occupy $A\left(\Gamma_{7}\right), B\left(\Gamma_{9}\right)$, and $C\left(\Gamma_{7}\right)$ valence bands. ${ }^{2-4}$ The three valence bands are split due to the crystal field and spin-orbit interactions. Usually, unintentionally doped $\mathrm{ZnO}$ is an $n$-type material due to a large amount of defects, most likely hydrogen impurity and Zn interstitials. ${ }^{1,4-7}$ Bound exciton complexes (BXs), i.e., excitons bound to neutral or ionized donors/acceptors, ${ }^{8,9}$ are easily formed at low temperature. Sharp BX lines dominate photoluminescence (PL) spectra of high quality $\mathrm{ZnO}$. Generally, most of $\mathrm{BX}$ transitions are attributed to $A$ excitons bound to neutral donor. ${ }^{4}$ This assumption is further used to study the top valence band symmetry of $\mathrm{ZnO}$. However, little experiment has been done to identify the electronic nature of BXs. In principle, both $A$ and $B$ excitons can be bound to defects due to the small splitting of $A$ and $B$ valence bands. Applying a magnetic field reveals the Zeeman splitting of BX's lines, and initially dipole forbidden transitions show up due to a lowering of the crystal symmetry. The splittings strongly depend on the nature of the defect center. ${ }^{10}$ Furthermore, by investigating the optical selection rules, which are uniquely determined by band symmetries, the type of excitons can be identified.

In this work, we study $\mathrm{BX}$ transitions of a bulk $\mathrm{ZnO}$ sample by magneto-PL. Ten sharp BX emissions are observed. We are able to classify the ten lines into three groups according to their magnetic field, angle, and circular polarization dependencies. The charge states of the defect centers

\footnotetext{
a) Present address: Laboratoire "Matériaux et Phénomènes Quantiques," Université Paris Diderot, Paris, France.

${ }^{b)}$ Present address: School of Physics \& Engineering, Sun Yat-sen University, Guangzhou, China.

c) Author to whom correspondence should be addressed. Electronic mail phjwang@ust.hk.
}

and the type of excitons are unambiguously identified and effective $g$ factors of electrons and holes are determined.

\section{EXPERIMENTAL DETAILS}

The sample investigated is an undoped melt-grown $\mathrm{ZnO}$ substrate grown along [0001] direction $(+c$ axis terminated by zinc, $n \sim 5 \times 10^{16} \mathrm{~cm}^{-3}$, thickness of $0.5 \mathrm{~mm}$, produced by Cermet Inc.). Magneto-PL measurements were carried out using a split-field superconducting magnetocryostat (Oxford Instrument Spectromag 4000). PL signals were collected and dispersed by a $0.85 \mathrm{~m}$ spectrometer (SPEX 1403) with 1800 $\mathrm{g} / \mathrm{mm}$ double gratings. The spectral resolution of the system is $\sim 0.1 \mathrm{meV}$. Faraday configuration $(\boldsymbol{B} \| \boldsymbol{k})$ was applied, where $\boldsymbol{k}$ is the wave vector of the emitted light. $\boldsymbol{B}$ is decomposed into an out-of-plane component $B_{\|_{c}}=B \cos \theta$ (parallel to the $c$-axis) and an in-plane component $B_{\perp c}=B \sin \theta$ (perpendicular to the $\boldsymbol{c}$-axis), while $\theta$ is the angle between $\boldsymbol{B}$ and the $\boldsymbol{c}$-axis [see Fig. 1(a) inset]. Different $\theta$ angles were achieved by simply rotating the $\boldsymbol{c}$-axis. Due to the poor signal to noise ratio for the edge emission of $\mathrm{ZnO}$ sample $(\theta$ $=90^{\circ}$ ), which causes a large error to find accurate peak positions, only data for $\theta$ up to $75^{\circ}$ are presented in this paper. The circular polarization $\left(\sigma_{+}\right.$and $\left.\sigma_{-}\right)$of emissions was analyzed using a wavelength-tunable quarter-wave plate and a linear polarizer. All the measurements were performed at $5 \mathrm{~K}$ to minimize energy shifts induced by thermal fluctuation.

\section{RESULTS AND DISCUSSION}

By investigating a near-band-edge PL spectrum at $5 \mathrm{~K}$, emissions of free excitons, bound excitons, two electron satellites, and LO phonon replicas were clearly observed. ${ }^{11} \mathrm{~A}$ high resolution PL spectrum of the dominant $\mathrm{BX}$ emissions taken at $5 \mathrm{~K}$ is shown as the top black curve in Fig. 1(a). In the range of 3.3568-3.3755 eV ten lines are resolved as indicated by arrows and labeled as 1-10. All lines are sharp and have a full width at half maximum of 0.1 to $0.3 \mathrm{meV}$, indicating a high quality of the sample. Considering the free $A$ exciton emission at $3.3778 \mathrm{eV}$ (Ref. 2) the binding ener- 


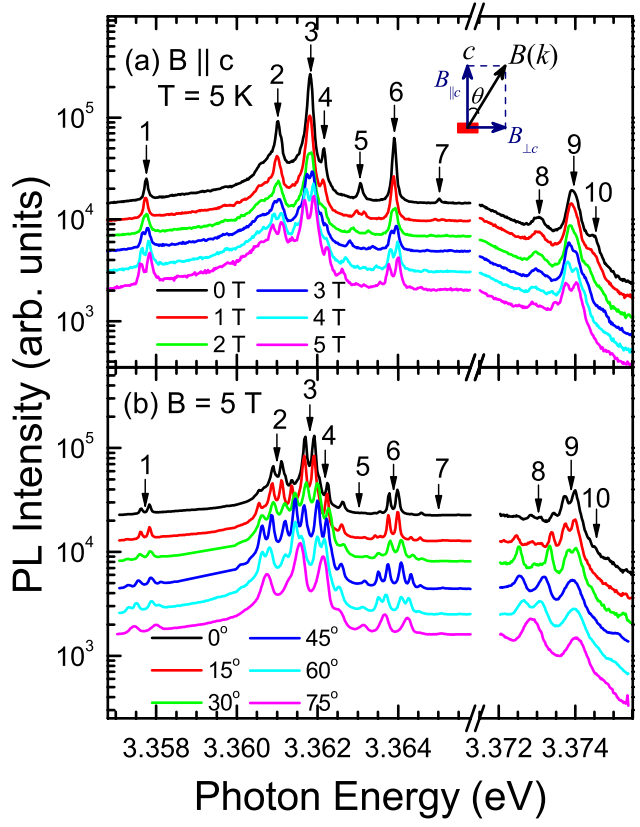

FIG. 1. (Color online) (a) The $\boldsymbol{B}$ field $(\boldsymbol{B} \| \boldsymbol{c})$ and (b) angle $\theta(B=5 \mathrm{~T})$ dependent magneto-PL spectra of BX emissions from 3.3568 to $3.3755 \mathrm{eV}$ at $T=5 \mathrm{~K}$. All spectra were vertically shifted for clarity.

gies of the BXs range from 3 to $20 \mathrm{meV}$, implying that the relevant defects are donorlike. ${ }^{4,12}$ The information of the ten BX lines are summarized in Table I. In comparison with the well-known exciton line $I_{0}$ to $I_{11}$ reported in earlier work ${ }^{12,15}$ some of BX lines in this work are identified accordingly and indicated in the table.

Figures 1(a) and 1(b) show magneto-PL spectra of BXs in dependence on the $\boldsymbol{B}$ field at $\boldsymbol{B} \| \boldsymbol{c}$ and angle $\theta$ at $B=5 \mathrm{~T}$, respectively. All spectra were vertically shifted for clarity. Diamagnetic shift in the BX lines is negligible indicating that they originate from $1 S$ excitons. ${ }^{13}$ From the dependences on the $\boldsymbol{B}$ field, angle $\theta$, and circular polarization of the split peaks, the ten lines are sorted into three groups. Group 1 consists of lines 1-4 and 6, group 2 of lines 8 and 9 , and group 3 of lines 5, 7, and 10 .

We plot in Fig. 2 the magneto-PL spectra of the three groups in a smaller spectral range. The evolution of peaks with respect to the $\boldsymbol{B}$ field and angle $\theta$ is traced by red dots.

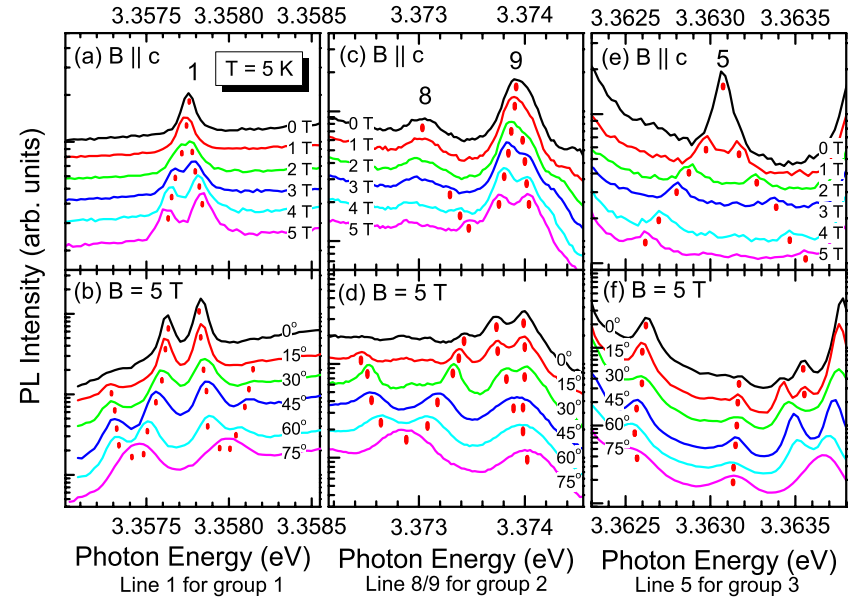

FIG. 2. (Color online) The magneto-PL spectra of the three groups in a smaller spectral range. (a) and (b) are for line 1 at $3.3577 \mathrm{eV}$ of group 1. (c) and (d) are for line 8 at $3.3730 \mathrm{eV}$ and line 9 at $3.3739 \mathrm{eV}$ of group 2. (e) and (f) are for line 5 at $3.3631 \mathrm{eV}$ of group 3.

Figures 2(a) and 2(b) show the Zeeman splitting of line 1 at $3.3577 \mathrm{eV}$ of group 1. At $\boldsymbol{B} \| \boldsymbol{c}$ it has a small Zeeman splitting, while two more peaks, outside of the split peaks at $\boldsymbol{B} \| \boldsymbol{c}$, appear at nonzero $\theta$. At an arbitrary $\theta$, line 1 splits into a quartet. In group 2, see Figs. 2(c) and 2(d), line 8 at 3.3730 $\mathrm{eV}$ has a larger Zeeman splitting and line 9 at $3.3739 \mathrm{eV}$ has a smaller splitting at $\boldsymbol{B} \| \boldsymbol{c}$. Both lines split into a doublet at an arbitrary $\theta$. Line 5 at $3.3631 \mathrm{eV}$ of group 3 shows a large Zeeman splitting at $\boldsymbol{B} \| \boldsymbol{c}$, which is comparable to the case of line 8. However, it has a different $\theta$ dependence [Figs. 2(e) and 2(f)]. Figures 3-5 summarize the peak energy dependences on $\mathrm{B}$ and $\theta$ of the selected lines of the three groups, respectively, which is more straightforward for further analyses.

Figure 3 shows the peak energies of line 1 at $3.3577 \mathrm{eV}$ with respect to $\boldsymbol{B}$ at (a) $\theta=0^{\circ}(\boldsymbol{B} \| \boldsymbol{c})$, (c) $\theta=60^{\circ}$, and to $\theta$ at (b) $B=5 \mathrm{~T}$. Circular polarizations $\left(\sigma_{+}\right.$and $\left.\sigma_{-}\right)$are indicated. We attribute it to a transition of a neutral donor bound $A$ exciton $\left(D^{0}, X^{A}\right){ }^{4}$ Figure $3(\mathrm{~d})$ shows a schematic transition of a $\left(D^{0}, X\right)$ complex. The ground state $\left(D^{0}\right)$ consists of a positive chemical center $(\oplus)$ (Ref. 14) and an electron $(-)$. The excited state $\left(D^{0} X\right)$ consists of $D^{0}$ and an electron/hole

TABLE I. A summary of the properties of the ten BX lines reported in the text including the wavelengths, transition energies, binding energies, $g$ factors of electrons and holes, and the bound exciton states. Lines with $*$ are identified according to Refs. 12 and 15 .

\begin{tabular}{cccccc}
\hline \hline Line & $\begin{array}{c}\lambda \\
(\mathrm{nm})\end{array}$ & $\begin{array}{c}\hbar \omega \\
(\mathrm{eV})\end{array}$ & $\begin{array}{c}\text { Exciton binding energy } \\
(\mathrm{meV})\end{array}$ & $g_{e} ; g_{h}^{\|} ; g_{h}^{\perp}$ & Bound exciton state \\
\hline $1^{*}\left(\mathrm{I}_{9}\right)$ & 369.300 & 3.3577 & 20.1 & $1.98 ;-1.36 ; 0.1$ & $D^{0}, X^{A}$ \\
$2^{*}\left(\mathrm{I}_{7}\right)$ & 368.938 & 3.3610 & 16.8 & $1.91 ;-1.03 ; 0.03$ & $D^{0}, X^{A}$ \\
$3^{*}\left(\mathrm{I}_{6}\right)$ & 368.850 & 3.3618 & 16.0 & $1.83 ;-0.99 ; 0.02$ & $D^{0}, X^{A}$ \\
$4^{*}\left(\mathrm{I}_{5}\right)$ & 368.806 & 3.3622 & 15.6 & $1.88 ;-1.13 ; 0.05$ & $D^{0}, X^{A}$ \\
5 & 368.707 & 3.3631 & 14.7 & $\ldots$ & unknown \\
$6^{*}\left(\mathrm{I}_{4}\right)$ & 368.620 & 3.3639 & 13.9 & $1.93 ;-1.34 ; 0.11$ & $D^{0}, X^{A}$ \\
7 & 368.499 & 3.3650 & 12.8 & $\ldots$ & unknown \\
$8^{*}\left(\mathrm{I}_{1}\right)$ & 367.625 & 3.3730 & 16.8 & $1.98 ;-1.18 ; \cdots$ & $D^{+}, X^{A}$ \\
$9^{*}\left(\mathrm{I}_{0}\right)$ & 367.527 & 3.3739 & 15.9 & $\cdots$ & unknown \\
10 & 367.473 & 3.3744 & 15.4 & & \\
\hline \hline
\end{tabular}




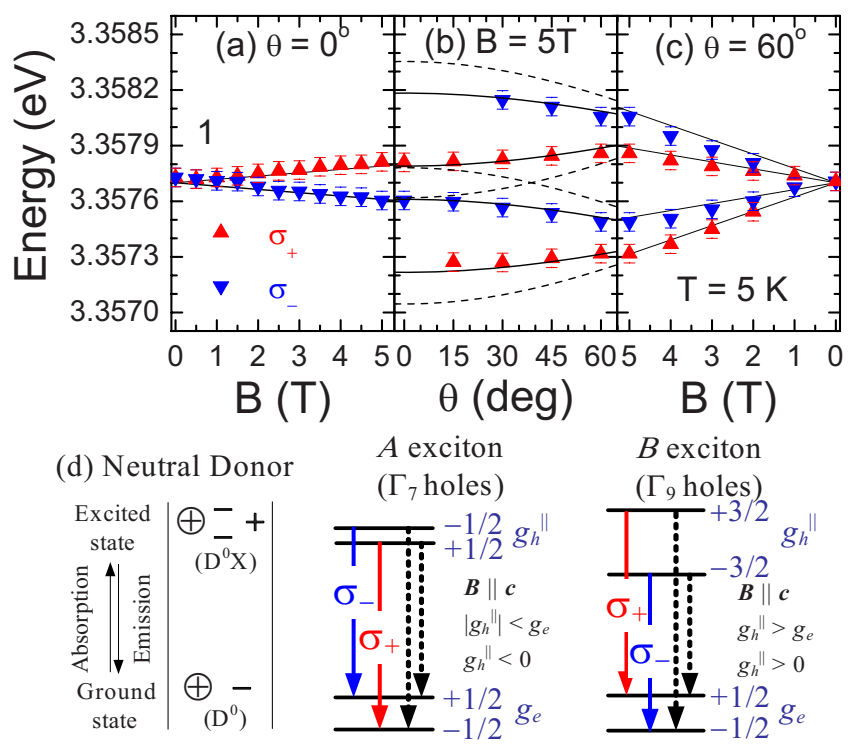

FIG. 3. (Color online) Zeeman splitting of line 1 as a function of $\boldsymbol{B}$ at (a) $\boldsymbol{B} \| \boldsymbol{c}$, (c) $\theta=60^{\circ}$, and (b) of $\theta$ at $B=5 \mathrm{~T}$. Solid curves are fittings for $A$ excitons, while dashed curves are for $B$ excitons. (d) Schematic energy diagrams of a neutral donor bound exciton $\left(D^{0} X\right)$. Splittings and transitions of $A$ and $B$ excitons at $\boldsymbol{B} \| \boldsymbol{c}$ are drawn separately. Solid and dashed arrows are for allowed and forbidden transitions, respectively.

$(-/+)$ pair $(X)$, in which two electrons are paired with antiparallel spins. Applying a $\boldsymbol{B}$ field, the ground and excited states have Zeeman splitting and the magnitude of the splitting is determined by the $g$ factor of electron $\left(g_{e}\right)$ for the ground state and of hole $\left(g_{h}\right)$ for the excited state, respectively. This gives inner-two dipole-allowed (solid arrows) and outer-two dipole-forbidden (dashed arrows) transitions. If $B_{\perp c} \neq 0$, the forbidden transitions become allowed.

Experimentally, line 1 splits into a doublet at $\boldsymbol{B} \| \boldsymbol{c}$ [Fig. 3(a)], which corresponds to allowed transitions. The upper Zeeman component has $\sigma_{+}$polarization. Two outer transitions appear for an arbitrary $\theta[$ Fig. 3(b)], which are forbid-

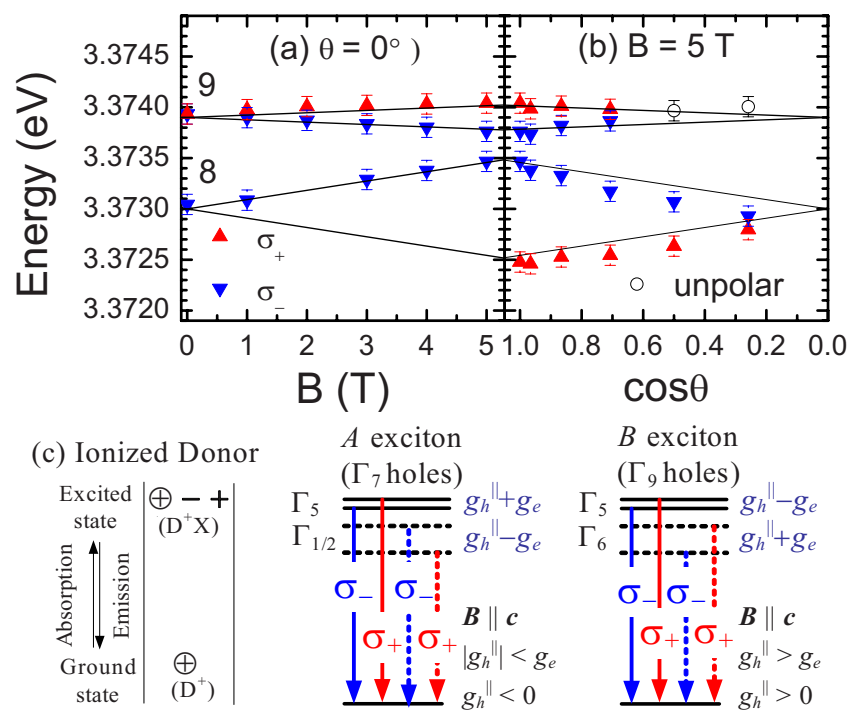

FIG. 4. (Color online) Zeeman splitting of the group 2 as a function of (a) $\boldsymbol{B}$ at $\boldsymbol{B} \| \boldsymbol{c}$ and (b) $\cos \theta$ at $B=5$ T. Lines are fittings. (c) Schematic energy diagrams of an ionized donor bound exciton $\left(D^{+} X\right)$. Splittings and transitions of $A$ and $B$ excitons at $\boldsymbol{B} \| \boldsymbol{c}$ are drawn separately. Solid and dashed arrows are for allowed and forbidden transitions, respectively.

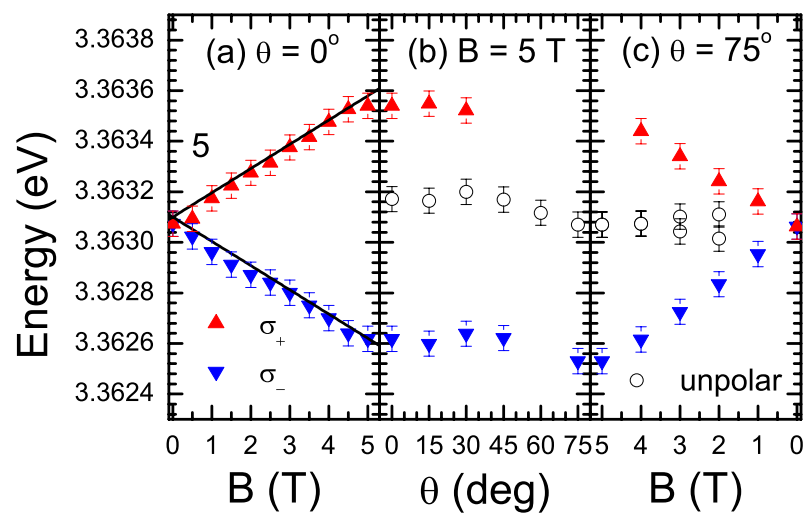

FIG. 5. (Color online) Zeeman splitting of line 5 at $3.3631 \mathrm{eV}$ as a function of $\boldsymbol{B}$ at (a) $\boldsymbol{B} \| \boldsymbol{c}$, (c) $\theta=75^{\circ}$, and of (b) $\theta$ at $B=5$ T. Lines are fittings $\Delta E$ $=g_{\text {exc }} \mu_{B} B$.

den at $\theta=0^{\circ}$. For $A$ excitons with $\Gamma_{7}$ holes, $\left|g_{h}^{\|}\right|<g_{e}$ and $g_{h}^{\|}$ $<0 .^{2-4}$ As a result [Fig. 3(d)], the dipole-allowed inner-two peak energies is given by $E_{L_{1}} \pm \frac{1}{2} \mu_{B} B\left(g_{e}+g_{h}\right)$, while the dipole-forbidden outer-two peaks by $E_{L_{1}} \pm \frac{1}{2} \mu_{B} B\left(g_{e}-g_{h}\right)$, where $\mu_{B}=5.788 \times 10^{-5} \mathrm{eV} \mathrm{T}^{-1}$ is the Bohr magneton, and $g_{h}=g_{h}^{\|} \sqrt{\cos ^{2} \theta+\left(g_{h}^{\perp} / g_{h}^{\|}\right)^{2} \sin ^{2} \theta}\left(g_{h}^{\|}\right.$for $\boldsymbol{B} \| \boldsymbol{c}$ and $g_{h}^{\perp}$ for $\boldsymbol{B} \perp \boldsymbol{c}$, $g_{h}^{\perp}$ is small ${ }^{4}$. Using $g_{e}=1.98, g_{h}^{\|}=-1.36$, and $g_{h}^{\perp}=0.1$, respectively, the data agree well with the fit [solid curves in Figs. 3(a)-3(c)]. It is worth mentioning that for $B$ excitons with $\Gamma_{9}$ holes, $g_{h}^{\|}>g_{e}>0$ and $g_{h}^{\perp}=0 .{ }^{4}$ The dipole-allowed inner-two peak energies are given by $E_{L_{1}} \pm \frac{1}{2} \mu_{B} B\left(g_{h}-g_{e}\right)$. It is straightforward to see that the two peaks should cross each other with increasing $\theta$ [see dashed curves in Fig. 3(b) with $g_{e}=1.98$ and $\left.g_{h}^{\|}=2.6\right]$. However, the crossing is not observed in our experiments. This further confirms our assignment that line 1 as well as other group 1 lines are associated with transitions of $A$ exciton but not $B$ exciton bound to neutral donors. The fittings of the modal to the data lead to $g_{e}$ $=1.83-1.98, g_{h}^{\|}=(-0.99)-(-1.36)$, and $g_{h}^{\perp}=0.02-0.11$ for $\mathrm{BX}$ lines in group 1. These values are similar to those reported in Ref. 4.

Figure 4 plots (a) the $\boldsymbol{B}$ field and (b) $\theta$ dependent split peaks of group 2 as well as the circular polarizations. We attribute line 8 at $3.3730 \mathrm{eV}$ and line 9 at $3.3739 \mathrm{eV}$ to paired transitions of an ionized donor bound $A$ exciton $\left(D^{+}, X^{A}\right)$, similar to $I_{2} / I_{3}$ reported in Ref. 4. Figure 4(c) shows a schematic transition of a $\left(D^{+}, X\right)$ complex. The ground state $\left(D^{+}\right)$ consists of the positive chemical center $(\oplus)$. The excited state $\left(D^{+} X\right)$ consists of $D^{+}$and an electron/hole $(-/+)$pair $(X)$. For $D^{+} X$, dipole-allowed (solid levels) and dipoleforbidden (dashed levels) exciton states split due to a zerofield exchange interaction induced by different combinations of electron and hole spins. By applying a $\boldsymbol{B}$ field, the ground state does not split but the two split excited states display Zeeman splitting. This gives the upper-two allowed (solid arrows) and lower-two forbidden (dashed arrows) transitions. The forbidden transitions can be activated if the laser is polarized along the $c$-axis. ${ }^{4}$

Experimentally, weak line 8 and strong line 9 indicate that the line 8 transition is forbidden, while line 9 is allowed at zero field. The energy separation between them is 0.9 $\mathrm{meV}$, which is in agreement with the reported electron-hole 
exchange energy. $3,4,15,16$ The polarization of the upper Zeeman component of line 9 is $\sigma_{+}$, while that of line 8 is $\sigma_{-}$. As shown in Fig. 4(c), for $A$ excitons, the allowed state has $\Gamma_{5}$ symmetry and antiparallel electron and hole spins with a small Zeeman splitting, whereas the forbidden state has $\Gamma_{1 / 2}$ symmetry and parallel electron and hole spins with a large Zeeman splitting. The polarization of upper Zeeman component of $\Gamma_{5}$ is $\sigma_{+}$and that of $\Gamma_{1 / 2}$ is $\sigma_{-}$, which agrees with our observation. The Zeeman splitting of lines $8 / 9$ can be fitted to $E_{L_{6}} \pm \frac{1}{2}\left(g_{h}^{\|}-g_{e}\right) \mu_{B} B_{\| c}$ and $E_{L_{7}} \pm \frac{1}{2}\left(g_{h}^{\|}+g_{e}\right) \mu_{B} B_{\| c}$, respectively. The electron and hole effective $g$ factors are obtained as $g_{e}=1.98$ and $g_{h}^{\|}=-1.18$ [solid curves in Figs. 4(a) and 4(b) ]. Considering the case of $B$ excitons, the polarizations of the upper Zeeman component of the allowed $\Gamma_{5}$ state and forbidden $\Gamma_{6}$ state are both $\sigma_{+}$. It is in contradiction to what we have observed.

Group 3 splits in a way that is different from two other groups. Figure 5 shows the splitting of line 5 at $3.3631 \mathrm{eV}$ as a function of the $\boldsymbol{B}$ field at (a) $\boldsymbol{B} \| \boldsymbol{c}$, (c) $\theta=75^{\circ}$, and of $\theta$ at (b) $B=5 \mathrm{~T}$. We note that the splitting at $\boldsymbol{B} \| \boldsymbol{c}\left(g_{\text {exc }}=3.11\right)$ is similar to that of the low energy component of an ionized donor BX. So, it is not a neutral defect BX transition. Although the upper splitting component has $\sigma_{+}$polarization, we cannot attribute it to the transition of exciton $B$ bound to an ionized defect, ${ }^{4}$ due to the lack of a paired higher-energy transition and little $\theta$ dependence of split peaks. In addition, two more peaks (indicated by black circles) appear in between the two split peaks at large $\theta$ and small $B$. In this work, so far, we cannot make a conclusive identification and further studies are needed.

\section{CONCLUSION}

In summary, our work provides a systematic study of the $\mathrm{BXs}$ of bulk $\mathrm{ZnO}$ using the magneto-PL spectroscopy. Ten $\mathrm{BX}$ lines are classified into three groups according to their $\boldsymbol{B}$ field, angle $\theta$, and circular polarization dependences. Group 1 is associated with the $1 S A$ exciton bound to neutral impurities, where $g_{e}=1.83-1.98, g_{h}^{\|}=(-0.99)-(-1.36)$, and $g_{h}^{\perp}$ $=0.02-0.11$. Group 2 corresponds to transitions of $\Gamma_{1 / 2}$ and $\Gamma_{5}$ excitons bound to ionized impurities, respectively, where $g_{e}=1.98$ and $g_{h}^{\|}=-1.18$. Our findings provide a clear identification of the origin of the sharp emission lines observed in high quality $\mathrm{ZnO}$ samples.

\section{ACKNOWLEDGMENTS}

The authors are grateful to the financial support of Research Grant Council of HKSAR via Grant Nos. 603704 and HKU 7056/06P.

${ }^{1}$ Ü. Özgür, Ya. I. Alivov, C. Liu, A. Teke, M. A. Reshchikov, S. Dogan, V. Avrutin, S.-J. Cho, and H. Morkoc, J. Appl. Phys. 98, 041301 (2005).

${ }^{2}$ L. Ding, C. L. Yang, H. T. He, F. Y. Jiang, J. N. Wang, Z. K. Tang, B. A. Foreman, and W. K. Ge, e-print arXiv:0706.3965.

${ }^{3}$ W. R. L. Lambrecht, A. V. Rodina, S. Limpijumnong, B. Segall, and B. K. Meyer, Phys. Rev. B 65, 075207 (2002).

${ }^{4}$ A. V. Rodina, M. Strassburg, M. Dworzak, U. Haboeck, A. Hoffmann, A. Zeuner, H. R. Alves, D. M. Hofmann, and B. K. Meyer, Phys. Rev. B 69, 125206 (2004).

${ }^{5}$ A. F. Kohan, G. Ceder, D. Morgan, and C. G. Van de Walle, Phys. Rev. B 61, 15019 (2000).

${ }^{6}$ A. Janotti and C. G. Van de Walle, Nature Mater. 6, 44 (2007).

${ }^{7}$ D. C. Look, G. C. Farlow, P. Reunchan, S. Limpijumnong, S. B. Zhang, and K. Nordlund, Phys. Rev. Lett. 95, 225502 (2005).

${ }^{8}$ M. A. Lampert, Phys. Rev. Lett. 1, 450 (1958).

${ }^{9}$ J. R. Haynes, Phys. Rev. Lett. 4, 361 (1960).

${ }^{10}$ D. G. Thomas and J. J. Hopfield, Phys. Rev. 128, 2135 (1962).

${ }^{11}$ S. J. Xu, S.-J. Xiong, and S. L. Shi, J. Chem. Phys. 123, 221105 (2005).

${ }^{12}$ B. K. Meyer, H. Alves, D. M. Hofmann, W. Kriegseis, D. Forster, F. Bertram, J. Christen, A. Hoffmann, M. Straßburg, M. Dworzak, U. Haboeck, and A. V. Rodina, Phys. Status Solidi B 241, 231 (2004).

${ }^{13}$ J. J. Hopfield and D. G. Thomas, Phys. Rev. 122, 35 (1961).

${ }^{14}$ D. G. Thomas and J. J. Hopfield, Phys. Rev. Lett. 7, 316 (1961).

${ }^{15}$ D. C. Reynolds, C. W. Litton, and T. C. Collins, Phys. Rev. 140, A1726 (1965).

${ }^{16}$ T. Skettrup, M. Suffczynski, and W. Gorzkowski, Phys. Rev. B 4, 512 (1971). 